[Video Article]

\section{New reports of confirmed pandiculation by spiders}

\author{
Shojiro Nagayama ${ }^{1}$ and Keizo Takasuka ${ }^{2 *}$ \\ ${ }^{I}$ Hamamatsu city, Shizuoka, Japan \\ E-mail:sasa100go@gmail.com \\ ${ }^{2}$ Institute for Advanced Biosciences, Keio University, \\ Yamagata, Japan \\ E-mail: keizaf@gmail.com, *Corresponding author
}

\begin{abstract}
Pandiculation within spiders has only recently been discovered - the first record of this habit among Protostomia - and it has only been demonstrated in one species (Cyclosa argenteoalba). We report here four further araneoid species i.e. Argiope bruennichi, Cyrtophora ikomosanensis, Trichonephila clavata (all Araneidae), and Nihonhimea japonica (Theridiidae) exhibiting pandiculation in or on their own webs. These records suggest that pandiculation is widely distributed at least among the superfamily Araneoidea, members of which generally construct aerial webs and wait in ambush for a long period.
\end{abstract}

Key words - Araneidae, Araneoidea, Protostomia, stretching out, Theridiidae

Pandiculation is the coordinated stretching and stiffening actions of the body as one unit (Fraser 1989), to arouse the voluntary cortex by making a strong voluntary muscle contraction to feed back an equally strong sensory stimulation to the motor neurons (Hanna 1990). It has been widely observed in Mammalia (Heusner 1946) and Aves (Hanna 1990), but never observed in other Vertebrata or at all in Invertebrata until 2021, where pandiculation-like behaviour was first observed in a spider (Cyclosa argenteoalba Bösenberg \& Strand 1906) (Takasuka 2021).
To discover whether this habit was more widespread amongst spiders, we recorded long span videos of several web-building araneoid spiders in or on their own webs in Hamamatsu city, Japan. In accordance with Takasuka (2021), we defined the movement of spiders stretching out all their legs straight under the prosoma as pandiculation. As a result, we filmed 22 examples of pandiculation performed by four species i.e. Argiope bruennichi (Scopoli 1772) (Fig. 1A, B), Cyrtophora ikomosanensis (Bösenberg \& Strand 1906) (Fig. 1C, D), Trichonephila clavata (L. Koch 1878) (Fig. 1E-G) (all Araneidae), and Nihonhimea japonica (Bösenberg \& Strand 1906) (Fig. 1H-J) (Theridiidae) (supplementary video). In all species, the pandiculation was confirmed in both females and males. In addition, a T. clavata spiderling flocking in a ball and a $N$. japonica spiderling inside a shelter made of dead leaves also practiced pandiculation (Fig. 1G, J).

These records suggest that pandiculation is widely distributed at least among the superfamily Araneoidea, members of which generally construct aerial webs and wait in ambush for a long period. We expect that future observations will also find widespread pandiculation performed across the RTA-clade and other ancestral spider groups.

\section{Acknowledgments}

We would like to express our cordial thanks to James F. Fleming (Oslo Natural History Museum) for kindly proofreading the manuscript.

\section{Data availability statement}

Digital video images (supplementary video) related to the article are available at https://doi.org/10.50997/data.asjaa.17139701

This movie is licensed under a Attribution-NonCommercial-No Derivatives 4.0 International (CC BY-NC-ND 4.0). To view a copy of this licence, visit https://creativecommons.org/licenses/by-nc-nd/4.0/

\section{References}

Fraser, A. F. 1989. Pandiculation: the comparative phenomenon of systematic stretching. Appl. Anim. Behav. Sci., 23: 263-268.

Hanna, T. 1990. Clinical somatic education: a new discipline in the field of health care. Somatics: Magazine-Journal of the Bodily Arts and Sciences, 8: 4-10.

Heusner, A. P. 1946. Yawning and associated phenomena. Physiol. Rev., 26: $156-168$.

Takasuka, K. 2021. Pandiculation documented in a spider. Front. Ecol. Environ., 19: 363-363.

Received October 27, 2021 / Accepted December 2, 2021 

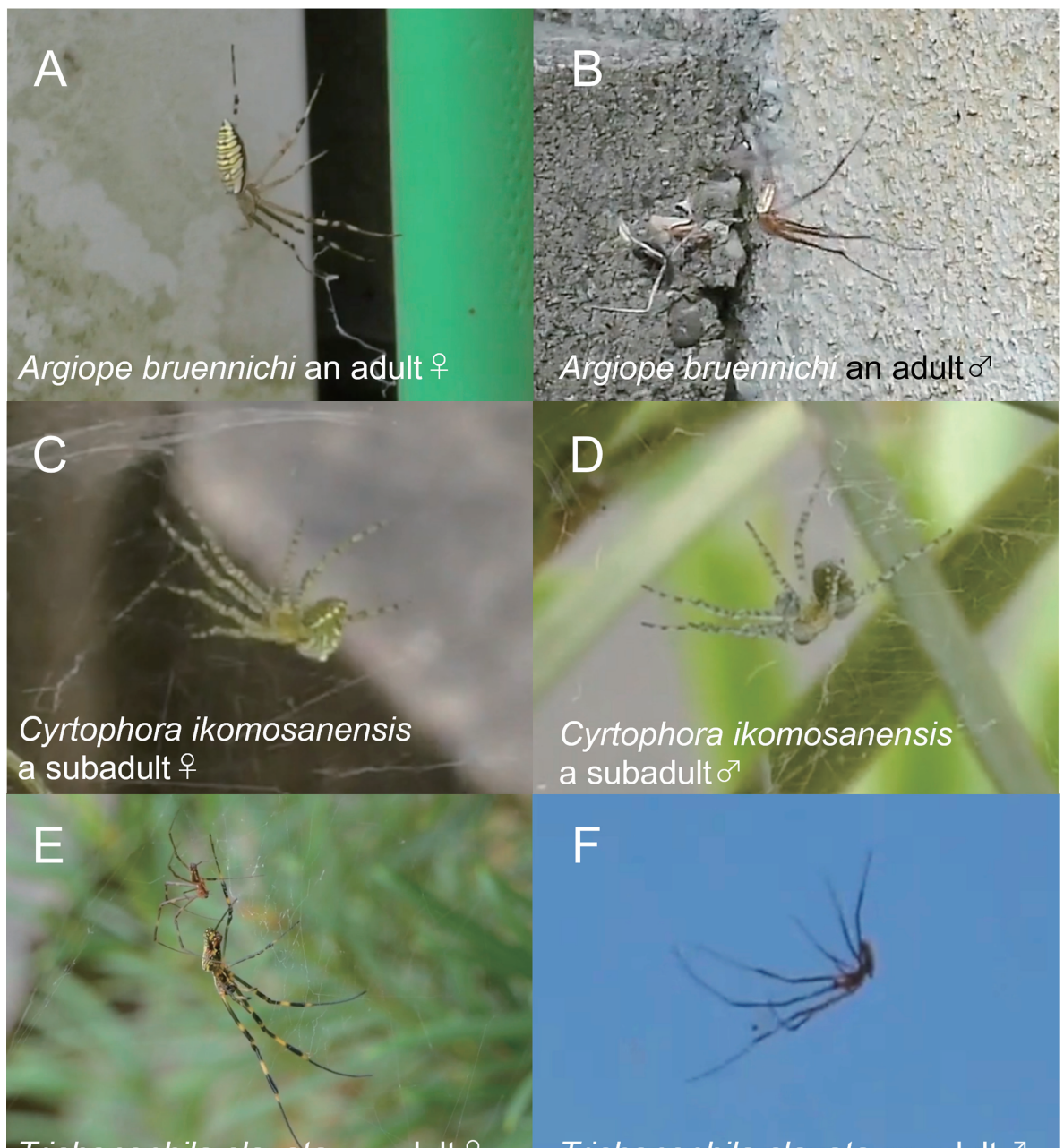

F

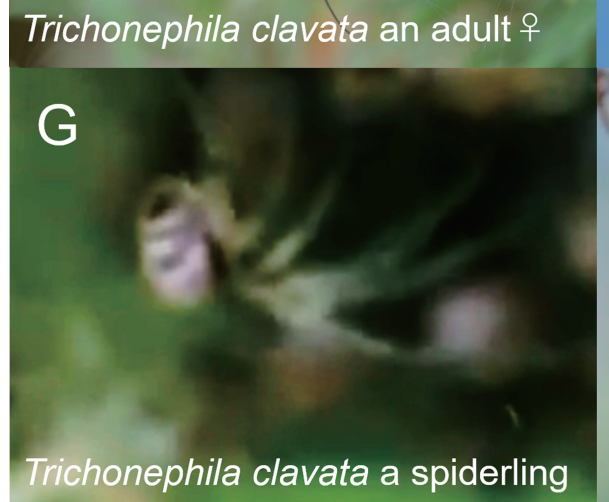

\section{Trichonephila clavata an adult ơ}
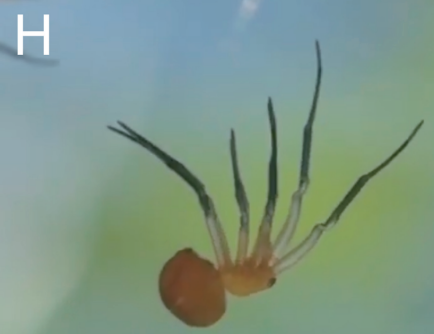

Nihonhimea japonica a subadult 우

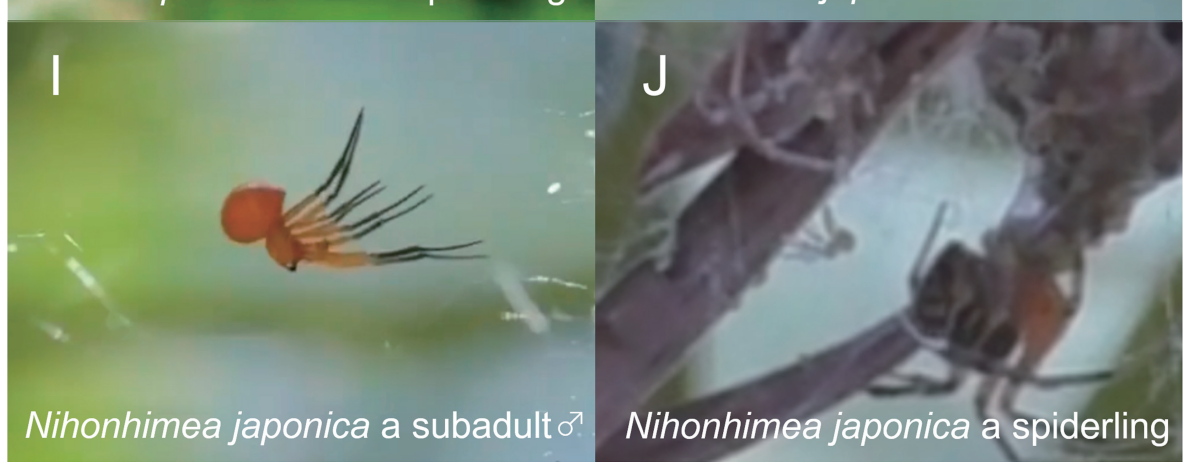

Fig. 1. Pandiculation by four araneoid species snapped by the videos. A-B, Argiope bruennichi (an adult female and an adult male, respectively); C-D, Cyrtophora ikomosanensis (a subadult female and a subadult male, respectively); E-G, Trichonephila clavata (an adult female, an adult male, and a spiderling, respectively); H-J, Nihonhimea japonica (a subadult female, a subadult male, and a spiderling, respectively). 